\title{
Pressão Arterial e Concentração Plasmática do Peptídeo Atrial Natriurético e do Peptídeo Natriurético Tipo B, em Gestações Complicadas pela Pré-eclâmpsia
}

\author{
Influence of Atrial Natriuretic Peptide and type B Natriuretic Peptide Plasma \\ Levels on Arterial Pressure in Pregnancies Complicated by Preeclampsia
}

Zilma Silveira Nogueira Reis, Antônio Carlos Vieira Cabral, Juliana Silva Barra, Henrique Vitor Leite, Alim Alves Demian, Adelina Martha dos Reis

\section{RESUM0}

\begin{abstract}
Objetivo: o estudo busca determinar a existência de associação entre a elevação da pressão arterial e os niveis plasmáticos dos peptídeos natriuréticos ANP e BNP, na gestação complicada pela pré-eclâmpsia.

Métodos: em estudo transversal caso-controle, pareado por idade gestacional, 25 grávidas normotensas e 61 portadoras de pré-eclâmpsia foram avaliadas quanto ao nível da pressão arterial e às concentrações plasmáticas dos peptídeos natriuréticos ANP e BNP. Exames clínico e laboratoriais foram realizados para o diagnóstico de pré-eclâmpsia, sendo a pressão arterial medida de forma não invasiva. As dosagens hormonais foram obtidas por radioimunoensaio, após extração em colunas Sep-pak C18. Os valores médios das concentrações plasmáticas do ANP e BNP foram comparados entre grupos com pressão arterial progressivamente maiores. A correlação entre os valores da pressão arterial e os níveis plasmáticos do ANP e BNP no sangue materno foi também investigada pela de análise de regressão no grupo completo de gestantes e em grupos específicos excluindo-se a hipertensão anterior à gestação e, em seguida, excluindo-se aquelas sem hipertensão prévia.

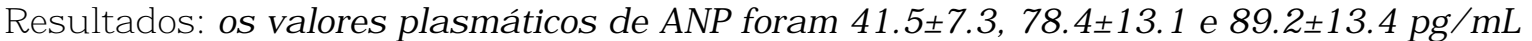
$(p<0,00001)$ e os de BNP plasmático foram $79.5 \pm 15.8,176.7 \pm 42.2$ e $208.3 \pm 63.5 \mathrm{pg} / \mathrm{mL}$ ( $p=0,005)$, respectivamente, para os grupos de pressão arterial média =107 mmHg, 107-139 $\mathrm{mmHg}$ e $=140 \mathrm{mmHg}$. Verificou-se correlação positiva entre as concentrações plasmáticas do ANP e os niveis pressóricos na pré-eclâmpsia, independente da existência de estado hipertensivo prévio à gestação ( $p<0,0001$ para pré-eclâmpsia e $p<0,01$ para pré-eclampsia sobreposta à hipertensão arterial crônica), ao passo que as dosagens de BNP não se mostraram associadas à pressão arterial no grupo com hipertensão arterial prévia à gestação $(p=0,004$ para pré-eclâmpsia e $p=0,18$ para pré-eclampsia sobreposta à hipertensão arterial crônica). Conclusão: o agravamento da hipertensão na pré-eclâmpsia correlacionou-se com as concentrações séricas do ANP e BNP, embora os valores do BNP possam ser influenciados pela existência de estado hipertensivo prévio.
\end{abstract}

PALAVRAS-CHAVE: Gestação. Pressão arterial. Pré-eclâmpsia. Peptídeo atrial natriurético. Peptídeo natriurético tipo $B$.

Correspondência:

Zilma Silveira Nogueira Reis

Centro de Medicina Fetal do Hospital das Clínicas da

Universidade Federal de Minas Gerais

Av. Alfredo Balena, 190, $4^{\circ}$ andar

30130-100 - Belo Horizonte-BH

Fone/FAX: (031) 3248-9421 / 9991-8256

zilma@hc.ufmg.br

\section{Introdução}

A pré-eclâmpsia é distúrbio multissistêmico cuja principal característica clínica é a hipertensão arterial. O estado hipertensivo é decorrente da vasoconstrição e muitas vezes se acompanha de 
complicações sistêmicas envolvendo o sistema nervoso central, cardiopulmonar, hepático e renal ${ }^{1}$.

$O$ peptídeo atrial natriurético (ANP) e peptídeo natriurético tipo $\mathrm{B}(\mathrm{BNP})$ pertencem à família dos peptídeos natriuréticos, sendo capazes de desencadear ações sobre o sistema circulatório como hipotensão, diurese, inibição da liberação e ação de vários hormônios incluindo a aldosterona, angiotensina II, endotelinas, renina e vasopressina ${ }^{2,3}$. Variações significativas nos níveis plasmáticos do ANP e do BNP, em função do estado gravídico, bem como de anormalidades hemodinâmicas da gestação como a hipertensão arterial, têm sido relatadas ${ }^{4}$. Em estudos experimentais, a secreção do ANP mostra-se correlacionada com a pressão arterial ${ }^{5,6}$ e pode ser experimentalmente verificada em resposta à distensão atrial induzida em ovelhas prenhes ${ }^{7}$.

Entretanto, a pré-eclâmpsia é condição única da gestação humana, o que dificulta modelos animais adequados para estudo de sua fisiopatologia. A associação entre a elevação da pressão arterial na pré-eclâmpsia e as concentrações plasmáticas do ANP e do BNP já tem sido evidenciada em alguns estudos ${ }^{8,9}$, mas que ainda não são conclusivos e, por vezes, são controversos ${ }^{10}$. Sendo assim, o papel da elevação pressórica na elevação do nível plasmático do ANP e BNP na gestação humana está ainda por ser determinado.

O presente estudo testa a hipótese de que a ascensão dos níveis da pressão arterial apresenta correlação com a elevação dos niveis séricos dos hormônios cardíacos ANP e BNP na gestação complicada pela pré-eclâmpsia, considerando-se a presença ou não de hipertensão crônica prévia.

\section{Pacientes e Métodos}

Para testar a hipótese de que a elevação da pressão arterial na gestação complicada pela préeclâmpsia é um dos determinantes da ascenção da concentração plasmática materna do ANP e $\mathrm{BNP}$, realizou-se estudo observacional transversal, do tipo caso-controle, pareado por idade gestacional. O estudo foi aprovado pelo Comitê de Ética em Pesquisa desta instituição.

No período de 10 meses, entre março de 2000 e janeiro de 2001, 25 grávidas normotensas e 61 hipertensas, entre a $28^{\mathrm{a}}$ e a $41^{\mathrm{a}}$ semana de gestação, foram selecionadas para o estudo. O grupo foi formado a partir de pacientes referenciadas para atendimento em nível terciário na Maternidade do Hospital das Clínicas da Universidade Federal de Minas Gerais, Brasil.

Foram critérios de inclusão a concordância com os termos do consentimento informado e o preenchimento dos critérios diagnósticos de préeclâmpsia do National High Blood Pressure Education Program Working Group on High Blood Pressure in Pregnancy $(2000)^{11}$. Os critérios são: sindrome ocorrendo após a $20^{\mathrm{a}}$ semana de gravidez, caracterizada pela elevação ou agravamento da hipertensão arterial e presença de proteinúria qualitativa positiva. Para o grupo controle, foram selecionadas pacientes normotensas, com pressão arterial inferior a 140/90 mmHg e pressão arterial média $<107 \mathrm{mmHg}$, pareadas pela idade gestacional às hipertensas. Estas foram escolhidas dentro do mesmo período e serviço, por apresentarem indicação para cesariana programada que não a hipertensão arterial.

Os critérios de exclusão, para ambos os grupos, foram a presença de sinais de trabalho de parto ou de condições que sabidamente interferem nos niveis circulantes destes hormônios, como as doenças renais, cardíacas ou diabete ${ }^{5,12}$.

As gestantes foram avaliadas durante a internação para resolução da gestação por elevação dos níveis pressóricos no grupo de hipertensas e para cesariana programada por outras indicações, no grupo controle. A pressão arterial foi medida no antebraço esquerdo, com a paciente na posição sentada por pelo menos cinco minutos, empregando-se pelo método auscultatório os sons I e V de Korotkoff. Tomou-se a média de pelo menos duas observações e a pressão arterial média (PAM) foi calculada pela fórmula $\mathrm{PAM}=2 / 3 \times$ pressão arterial diastólica $+1 / 3$ pressão arterial sistólica.

Para dosagem dos peptídeos no plasma materno, foi colhida amostra com cerca de $10 \mathrm{~mL}$ de sangue por meio da punção da veia antecubital, com a paciente na posição sentada. Este material foi obtido após a verificação da pressão arterial e antes de a gestante ser encaminhada ao bloco obstétrico, empregando-se sistema a vácuo e tubos contendo citrato. As gestantes não estavam recebendo fluidoterapia venosa no momento da coleta sanguínea e o horário do procedimento foi considerado.

A separação do plasma seguiu-se à coleta, sendo feita por centrifugação, por um período de 10 minutos, empregando-se a velocidade de 4000 rotações por minuto (400.000 g), a quatro graus centígrados. O material foi armazenado em congelador $\mathrm{a}-80^{\circ} \mathrm{C}$.

A técnica de dosagem empregada, o radioimunoensaio de duplo anticorpo, foi antecedida de extração em colunas Sep-Pak C $18{ }^{\circledR}$ (Waters Associates, Milford, MA, USA). A solução extraída sofreu processo de secagem por evaporação, em equipamento Speed-Vac Eppendorf ${ }^{\circledR}$, sendo mantida $\mathrm{a}-80^{\circ} \mathrm{C}$ até o dia do ensaio. A iodação do ANP humano (Ser 99-Tyr 126 marca Península Laborato- 
ries Inc., Belmont, CA, USA) foi realizada pelo método da lactoperoxidase ${ }^{12}$ com I ${ }^{125}$-sódico.

No dia do ensaio, as amostras foram reconstituídas com tampão (fosfato de sódio $0,1 \mathrm{M}$, $\mathrm{NaCl} 0,14 \mathrm{M}$, albumina bovina $0,1 \%$, azida sódica 0,01\%, Triton X-100 0,1\%, pH 7,4). O ANP foi reconstituído com $500 \mu \mathrm{L}$ desse tampão e o BNP com $250 \mu \mathrm{L}$. O ANP e o BNP imunorreativos foram dosados por radioimunoensaio em experimentos separados. Foram obtidas 81 dosagens de ANP e 75 de BNP, uma vez que parte das amostras foi empregada para padronização do ensaio.

Os valores médios das concentrações plasmáticas do ANP e BNP foram comparados por meio do teste $t$ de médias, entre grupos com pressões arteriais médias (PAM) progressivamente maiores (PAM $\leq 107$ mmHg, 107-139 mmHg e $\geq 140$ $\mathrm{mmHg}$ ). A relação de dependência entre as variáveis contínuas pressão arterial e nivel plasmático de cada peptídeo natriurético foi verificada por meio da regressão dos mínimos quadrados e análise de variância. A adequação do modelo foi avaliada pelo teste de hipótese HO: $\beta=0$, com o cálculo do intervalo de confiança (IC), empregando-se o programa Minitab Inc ${ }^{\circledR}$. versão 11.12 de 1996 . O nível de significância aceitável para os testes de hipótese foi $\mathrm{p}<0,05$.

\section{Resultados}

As principais características dos grupos estudados são mostradas na Tabela 1. Nota-se que o grupo de grávidas portadoras de pré-eclâmpsia apresentou idade materna, idade gestacional, raça e horário da coleta semelhantes às normotensas. No entanto, as gestantes portadoras de préeclâmpsia apresentaram menor paridade e nivel mais elevado de pressão arterial, como esperado.

Os valores plasmáticos médios do ANP e do BNP são mostrados na Tabela 2 para grupos de PAM $\leq 107 \mathrm{mmHg}, 107-139 \mathrm{mmHg}$ e $\geq 140 \mathrm{mmHg}$. Observaram-se concentrações médias crescentes destes peptídeos nos grupos com valores de pressão arterial cada vez maiores.

Tabela 1 - Características clínicas das grávidas incluídas no estudo.

\begin{tabular}{|c|c|c|c|}
\hline & $\begin{array}{c}\text { Hipertensas } \\
n=61\end{array}$ & $\begin{array}{l}\text { Normotensas } \\
\qquad n=25\end{array}$ & $\mathrm{p}$ \\
\hline Pressão arterial média $(\mathrm{mmHg}) \pm \mathrm{DP}$ & $133,8 \pm 17,7$ & $89,6 \pm 9,6$ & $<0,0001$ \\
\hline Hipertensão arterial prévia & $22(36,1 \%)$ & $\ldots$ & \\
\hline Uso de vasodilatadores & $32(52,5 \%)$ & $\ldots$ & \\
\hline Idade materna (anos) $\pm \mathrm{DP}$ & $27,5 \pm 6,1$ & $29,9 \pm 6,1$ & 0,07 (NS) \\
\hline Paridade $\pm \mathrm{DP}$ & $2,2 \pm 1,7$ & $3,4 \pm 2,5$ & $0,04(S)$ \\
\hline Raça: branca & 17 & 6 & \\
\hline Raça: não branca & 45 & 19 & 0,74 (NS) \\
\hline Idade gestacional (semanas) $\pm \mathrm{DP}$ & $34,8 \pm 3,5$ & $36,5 \pm 3,9$ & $0,06(\mathrm{NS})$ \\
\hline Horário da coleta sanguínea \pm DP (fração do dia) & $0,57 \pm 0,27$ & $0,54 \pm 0,13$ & $0,48(\mathrm{NS})$ \\
\hline
\end{tabular}

S: significativo, $p<0,05$; NS: não significativo DP: desvio-padrão

Tabela 2 - Concentração plasmática do ANP e BNP nos grupos de pacientes divididas conforme o nível de hipertensão.

\begin{tabular}{|c|c|c|c|c|}
\hline & $\begin{array}{c}\text { Grupo } 1 \\
n=28\end{array}$ & $\begin{array}{c}\text { Grupo } 2 \\
n=37\end{array}$ & $\begin{array}{c}\text { Grupo } 3 \\
n=21\end{array}$ & $p\left(^{*}\right)$ \\
\hline Pressão arterial média \pm EP (mmHg) & $88,8 \pm 1,67$ & $125,7 \pm 1,2$ & $152,6 \pm 2,8$ & $<0,0001$ \\
\hline ANP plasmático \pm EP (pg/mL) & $41,4 \pm 7,3$ & $78,4 \pm 13,1$ & $89,2 \pm 13,4$ & $<0,0001$ \\
\hline BNP plasmático \pm EP $(\mathrm{pg} / \mathrm{mL})$ & $79,5 \pm 15,8$ & $176,7 \pm 42,2$ & $208,3 \pm 63,5$ & 0,005 \\
\hline
\end{tabular}

$\left(^{\star}\right)$ Test $t$ de médias; EP: erro padrão

Grupo 1: Pressão arterial média = $107 \mathrm{mmHg}$; grupo 2: pressão arterial média 107-139 mmHg; grupo 3: pressão arterial média =140 mmHg.

As concentrações plasmáticas de ANP, tomadas de forma contínua, foram também avaliadas em função de valores ascendentes da pressão arterial. Equações de regressão foram obtidas, considerando-se o efeito da pressão arterial (variável preditora) sobre o nível plasmático do ANP (variá- vel resposta). As pressões arteriais sistólica, diastólica e média se correlacionaram diretamente à concentração plasmática do ANP $\left(\log _{10}\right.$ ANP = $1.10+0.00382 \mathrm{PAS}, \mathrm{p}<0,001 ; \log _{10} \mathrm{ANP}=1.15+$ $0.00527 \mathrm{PAD}, \mathrm{p}<0,001 ; \log _{10} \mathrm{ANP}=1.10+$ 0.0049PAM, p<0,001). 
Análise semelhante foi realizada entre a pressão arterial e as concentrações plasmáticas de BNP. Observou-se que a ascensão da pressão arterial também determinou modificação significativa nos valores do $\mathrm{BNP}$ sérico $\left(\log _{10} \mathrm{BNP}=1.41+\right.$ 0.0035PAS, $\mathrm{p}=0,014 ; \log _{10} \mathrm{BNP}=1.50+0.0044 \mathrm{PAD}$, $\mathrm{p}=0,027 ; \log _{10} \mathrm{BNP}=1.44+0.0043 \mathrm{PAM}, \mathrm{p}=0,018$ ).

Entre as pacientes com diagnóstico de préeclâmpsia, 39 (63,9\%) não apresentavam histórico nem evidências clínicas ou laboratoriais de hipertensão arterial crônica anterior à gestação, ao passo que as demais $22(36,1 \%)$ já eram portadoras de doença hipertensiva. Tomando-se 64 casos dentre os quais foram excluídas da análise as pacientes com hipertensão arterial prévia à gestação, observou-se presença de correlação entre a PAM e as concentrações plasmáticas dos peptídeos ANP $\left(\log _{10}\right.$ ANP $\left.=0.899+0.0070 P A M,<0,0001\right)$ e BNP $\left(\log _{10} \mathrm{BNP}=1.20+0.0067 \mathrm{PAM}, \mathrm{p}=0,004\right)$.

Quando são avaliados separadamente os 48 casos dos quais foram excluídas as gestantes sem hipertensão arterial prévia observa-se que apenas o ANP apresenta-se correlacionado à elevação da PAM $\left(\log _{10} \mathrm{ANP}=1.14+0.0039 \mathrm{PAM}, \mathrm{p}=0,01\right)$. Esta análise mostra que na presença de hipertensão prévia a elevação da pressão arterial não determina aumento proporcional na concentração plasmática do BNP $\left(\log _{10} \mathrm{BNP}=1.56+0.0024 \mathrm{PAM}, \mathrm{p}=0,18\right)$.

\section{Discussão}

A motivação pela busca de relações entre a pré-eclâmpsia e o sistema dos peptídeos natriuréticos fundamenta-se nas diversas dúvidas que ainda envolvem a fisiopatologia da préeclâmpsia e a verdadeira contribuição dos peptídeos vasoativos nessa doença.

A relevância do ANP e do BNP no balanço homeostático dos líquidos corporais e na regulação da pressão arterial vem sendo ressaltada desde sua descoberta ${ }^{2,6}$, pois, acredita-se que suas ações biológicas, particularmente seu efeito natriurético e vasodilatador, sejam compatíveis com uma participação de importância na regulação da pressão sanguínea.

Os grupos e subgrupos apresentados neste estudo seguiram a orientação quanto à classificação dos distúrbios hipertensivos da gravidez recomendada pelo National High Blood Pressure Education Program Working Group on High Blood Pressure in Pregnancy (2000) ${ }^{11}$ que serviu de forma especial para diferenciação entre pré-eclâmpsia e pré-eclâmpsia sobreposta a hipertensão crônica, pois permitiu o estudo de gestantes hipertensas com fisiopatologias diferenciadas, o que influiu marcadamente nas relações entre o peptídeo vasoativo BNP e a pressão arterial.

Ficou claro que o ANP possui, pelo menos em parte, sua concentração plasmática relacionada à elevação do nível pressórico, fato evidenciado pela correlação positiva entre estes parâmetros, independemente do tipo de doença hipertensiva. Tal achado é compativel com o estímulo da sobrecarga pressórica sobre as câmaras cardíacas determinando sintese e liberação do ANP, como sugerem vários autores ${ }^{13-16}$. O ANP apresentou correlação direta com os valores da pressão arterial, mesmo na presença de doença hipertensiva prévia, o que nos leva a crer que a liberação desse hormônio tenha sido estimulada pelo agravamento da hipertensão.

Em relação ao BNP, observou-se ausência de associação significativa com a elevação da pressão arterial determinada pela pré-eclâmpsia sobreposta à doença hipertensiva crônica, embora a associação esteja presente quando se considera grupo completo de gestante com hipertensão. Verificou-se que a associação direta com a elevação da hipertensão arterial foi evidenciada exclusivamente nas portadoras de pré-eclâmpsia isolada.

Dados a respeito do BNP na hipertensão gestacional são limitados, mas sabe-se que, distintamente do ANP, ele se associa melhor aos indicadores de gravidade do quadro hipertensivo, não avaliados neste estudo, como a hipertrofia ventricular esquerda ${ }^{17}$. Embora o subgrupo de gestantes com pré-eclâmpsia sobreposta à hipertensão crônica venha sendo excluído dos estudos clínicos, supõe-se que esta observação esteja associada a existência de niveis já elevados deste hormônio pela hipertensão crônica. Também é possivel a existência de limiar de resposta no mecanismo de distensão arterial levando a síntese e degranulação do BNP no qual a proporcionalidade dessa relação deixaria de existir, ou outra hipótese seria a ação de mecanismos contra-regulatórios na sua liberação. Este foi um resultado importante, pois não havia sido ainda obtido da forma que foi apresentado neste estudo.

Verifica-se também haver coerência entre nossos achados e a relação de causalidade entre a elevação do ANP secundária à distensão das câmaras cardíacas, pois a elevação da pressão arterial no grupo completo de pacientes estaria refletindo a sobrecarga pressórica sobre o coração, uma vez que a pré-eclâmpsia é estado reconhecidamente hipovolêmico ${ }^{18}$. Observação semelhante foi relatada por Pouta et al. ${ }^{16}$, que atribuem à vasoconstrição a responsabilidade de sobrecarga volumétrica localizada no átrio cardíaco capaz de causar aumento na liberação de ANP para a circulação sanguínea. 
Por outro lado, o BNP, ao se correlacionar exclusivamente com a pressão arterial no subgrupo da pré-eclâmpsia isolada, mostra que provavelmente estímulos para secreção são devido à sobrecarga aguda exibida pelo ventrículo esquerdo nas gestantes sem doença hipertensiva prévia, como sugerido por Nissel et al. ${ }^{19}$. Esses autores verificaram espessamento na parede ventricular posterior esquerda na diástole e no septo interventricular na sístole, em pacientes pré-eclâmpticas, independentemente do curto período de duração da hipertensão. Essa rápida subida da pressão seria decorrente do aumento na póscarga ocasionado pela elevação da resistência vascular periférica.

Ressalta-se, ainda, que o comportamento diferenciado entre o ANP e o BNP encontra também subsídios no fato de que, embora semelhantes em alguns aspectos como na estrutura molecular, mecanismo de síntese e forma circulante, há diferencial nos sítios de produção, expressão gênica e metabolismo ${ }^{5}$. Essas características coincidentes e discordantes já são reconhecidas desde a descoberta do $\mathrm{BNP}^{20}$, o que atribui aos peptídeos natriuréticos a característica de um sistema hormonal duplo, com mecanismos regulatórios diferenciados ${ }^{5,20}$. Tem sido relatado que a liberação e síntese do ANP são primariamente reguladas pelo estiramento atrial, ao passo que o BNP reflete melhor a massa e sobrecarga ventricular ${ }^{21}$.

Desta forma, é possivel que, à semelhança do que tem sido mostrado em outros estados patológicos do coração ${ }^{21}$, o BNP seja marcador importante da função cardíaca também na préeclâmpsia, o que, no entanto, não foi objeto deste trabalho. Recentemente, Borghi et al. ${ }^{22}$ mostraram que as gestantes pré-eclâmpticas apresentam adaptações cardíacas à elevação pressórica, com significante modificação na estrutura e na função do ventrículo esquerdo e que esses achados se relacionam com os niveis plasmáticos do ANP e BNP.

A razão para a elevação dos níveis circulantes dos peptídeos natriuréticos ANP e BNP na pré-eclâmpsia não foi ainda totalmente esclarecida. Vários fatores não são apontados: a diminuição da complacência do leito vascular determinada pela vasoconstrição ${ }^{19}$; o decréscimo do número de receptores $\mathrm{C}$ nos rins e em todo endotélio vascular ${ }^{23}$; o comprometimento da função renal $^{24}$ nessa doença e, finalmente, a lesão endotelial que tem papel central na préeclâmpsia ${ }^{25}$. Entretanto, a complexidade do mecanismo fisiopatológico da doença e da própria regulação hemodinâmica humana, especialmente na gestação, dificulta o esclarecimento dos fa- tores determinantes desta elevação, considerada paradoxal por alguns.

Este estudo contribui para a evidenciação de uma relação de dependência entre a elevação da pressão sanguínea na gestação e a ascensão do ANP e BNP nos grupos específicos anteriormente discutidos. No que se refere aos niveis circulantes do ANP, esta elevação seria decorrente da elevação da pressão arterial pela sobrecarga aguda ou pelo agravamento da sobrecarga crônica. Em relação ao BNP, a presença de estado hipertensivo prévio precisaria ser considerado para correta interpretação de seus níveis circulantes. Uma provável perspectiva no emprego das dosagens séricas dos peptídeos natriuréticos na pré-eclampsia é a de que elas possam ser um marcador de gravidade e prognóstico da doença que cursa com sobrecarga cardíaca, fato já verificado anteriormente em outras situações mórbidas ${ }^{3,21}$.

\section{ABSTRACT}

Purpose: to determine the existence of association between blood pressure rise and plasma ANP and BNP levels in pregnancies complicated by preeclampsia, considering the existence of a hypertensive state before pregnancy and supportive drug influence on these hormones.

Methods: in a case-control transversal study, 86 pregnant women were assessed regarding arterial pressure level and plasma ANP and BNP levels. Clinical and laboratory tests were carried out to diagnose preeclampsia and the use of hypotensive drugs and magnesium sulfate was considered. Hormone determinations were obtained through radioimmunoassay, after extraction in C18 Sep-pak columns. Correlation was investigated by means and regression analysis in the whole group of pregnant women and in specific groups, considering prior hypertension.

Results: plasma ANP values were 41.5 $\pm 7.3,78.4 \pm 13.1$ and $89.2 \pm 13.4 \mathrm{pg} / \mathrm{mL}(p<0.00001)$ and plasma BNP values were $79.5 \pm 15.8,176.7 \pm 42.2$ and $208.3 \pm 63.5 \mathrm{pg} / \mathrm{mL}(p=0.005)$, respectively, for mean blood pressure $=107 \mathrm{mmHg}, 107-139$ $\mathrm{mmHg}$ and $=140 \mathrm{mmHg}$. It was verified that the positive correlation between plasma ANP concentrations and pressure levels in preeclampsia did not depend on the existence of a hypertensive state before pregnancy ( $p<0.0001$ : preeclampsia and $p<0.01$ : preeclampsia superimposed on chronic hypertension), whereas BNP dosages were not associated with the arterial pressure in the group with arterial hypertension prior to pregnancy ( $p=0.004$ : preeclampsia and $p=0.18$ : preeclampsia superimposed on chronic hypertension).

Conclusion: aggravation of hypertension in preeclampsia correlates with serum ANP and BNP concentrations, although BNP values may be influenced by the existence of a prior hypertensive state.

KEYWORDS: Pregnancy. Blood pressure. Preeclampsia. Atrial natriuretic peptideltype $B$ natriuretic peptide. 


\section{Referências}

1. Groenendijk R, Trimbos JB, Wallenburg HC. Hemodynamic measurements in preeclampsia: preliminary observations. Am J Obstet Gynecol 1984; 150:232-6.

2. de Bold AJ, Borenstein HB, Veress AT, Sonnenberg H. A Rapid and potent response to intravenous injection of atrial myocardial extract in rats. Life Sci $1981 ; 28: 89-94$.

3. Mukoyama M, Nakao K, Hosoda K, et al. Brain natriuretic peptide as a novel cardiac hormone in humans. Evidence for an exquisite dual natriuretic peptide system, atrial natriuretic peptide and brain natriuretic peptide. J Clin Invest 1991; 87:1402-12.

4. Castro LC, Hobel CJ, Gornbein J. Plasma levels of atrial natriuretic peptide in normal and hypertensive pregnancies: a meta-analysis. Am J Obstet Gynecol 1994; 171:1642-51.

5. Ruskoaho H. Atrial natriuretic peptide: synthesis, release, and metabolism. Pharmacol Rev 1992; 44:479-602.

6. Morii N, Nakao K, Kihara M, et al. Decreased content in left atrium and increased plasma concentration of atrial natriuretic polypeptide in spontaneous hypertensive rats (SHR) and SHR stroke-prone. Biochem Biophys Res Commun 1986; 135:74-81.

7. Javeshghani D, Mukaddam-Daher S, Fan L, et al. Control of atrial natriuretic peptide factor by right and left distension in pregnant sheep. Am J Physiol 1995; 268:R1411-7.

8. Pouta AM, Karinen J, Vuolteenaho O, Laatikainen T. Pre-eclampsia: the effect of intravenous fluid preload on atrial natriuretic peptide during caesarean section under spinal anesthesia. Acta Anaesthesiol Scand 1996; 40:1203-9.

9. Itoh H, Sagawa N, Hasegawa M, et al. Brain natriuretic peptide is present in the human amniotic fluid and is secreted from amnion cells. J Clin Endocrinol Metab 1993; 76:907-11.

10.Mikkelsen AL, Schutten G, Asping U, Schutten HJ. Plasma concentration of atrial natriuretic peptide in normal pregnant women and in pregnant women with preeclampsia. Gynecol Obstet Invest 1991; 31:192-5.

11.Report of the National High Blood Pressure Education Program Working Group on High Blood Pressure in Pregnancy. Am J Obstet Gynecol 2000; 183:S1-S22.

12.Gutkoswska J, Antunes-Rodrigues J, McCann SM. Atrial natriuretic peptide and pituitary gland. Physiol Rev 1997; 77:465-515.

13. Metzler $\mathrm{CH}$, Lee ME, Thrasher TN, Ramsay DJ. Increased right or left atrial pressure stimulates release of atrial natriuretic peptides in conscious dogs. Endocrinology 1986; 119:2396-8.
14. Miyamoto S, Shimokawa H, Sumioki H, Touno A Nakano H. Circadian rhythm of plasma atrial natriuretic peptide, aldosterone, and blood pressure during the third trimester in normal and preeclamptic pregnancies. Am J Obstet Gynecol 1988; 158:393-9.

15.Merkouris RW, Miller FC, Catanzarite V, Quirk JG Jr, Rigg LA, Vesely DL. The N-terminal and Cterminal portions of the atrial natriuretic factor prohormone increased during preeclampsia. Am J Obstet Gynecol 1991; 164:1197-202.

16.Pouta AM, Rasanen JP, Airaksinen KE, Vuolteenaho OJ, Laatikainen TJ. Changes in maternal heart dimensions and plasma atrial natriuretic peptide levels in the early puerperium of normal and preeclamptic pregnancies. Br J Obstet Gynaecol 1996; 103:988-92.

17. Richards AM. The natriuretic peptides and hypertension. J Intern Med 1994; 235:543-60.

18. Silver HM, Seebeck M, Carlson R. Comparison of total blood volume in normal, preeclamptic, and nonproteinuric gestational hypertensive pregnancy by simultaneous measurement of red blood cell and plasma volumes. Am J Obstet Gynecol 1998; 179:87-93.

19.Nissel H, Carlstrom K, Cizinsky S, Grunewald C, Nylund L, Randmaa I. Atrial natriuretic peptide concentrations and hemodynamic effects of acute plasma volume expansion in normal pregnancy and preeclampsia. Obstet Gynecol 1992; 79:902-7.

20.Sudoh T, Kangawa K, Minamino N, Matsuo H. A new natriuretic peptide in porcine brain. Nature 1988; 332:78-81.

21.Omland T, Aakvaag A, Bonarjee VV, et al. Plasma brain natriuretic peptide as an indicator of left ventricular systolic function and long-term survival after acute myocardial infarction. Comparison with plasma atrial natriuretic peptide and N-terminal proatrial natriuretic peptide. Circulation 1996 93:1963-9.

22.Borghi C, Esposti, DD, Immordino V, et al. Relationship of systemic hemodynamics, left ventricular structure and function, and plasma natriuretic peptide concentrations during pregnancy complicated by preeclampsia. Am J Obstet Gynecol 2000; 183:140-7.

23.Kishimoto I, Nakao K, Suga S, et al. Down regulation of C-receptor by natriuretic peptides via ANP-B receptor in vascular smooth muscle cells. Am J Physiol 1993; 265:H1373-9.

24.Irons DW, Baylis PH, Butler TJ, Davison JM. Atrial natriuretic peptide in preeclampsia: metabolic clearance, sodium excretion and renal hemodynamics. Am J Physiol 1997; 273:F483-7.

25.Friedman SA, Schiff E, Emeis JJ, Dekker GA, Sibai BM. Biochemical corroboration of endothelial involvement in severe preeclampsia. Am J Obstet Gynecol 1995; 172:202-3.

Recebido em: 11/7/2003 Aceito com modificações em: 24/7/2003 